\title{
CHRONIC EXPOSURE TO BISPHENOL A PRODUCES MORPHOLOGICAL DERANGEMENTS IN LIVER, KIDNEY AND HEART IN RATS
}

\author{
Mahendra K. Pant ${ }^{\star}$, Jayanti Pant ${ }^{\star \star}$, Shripad B. Deshpande ${ }^{\star * *}$ \\ *Department of Anatomy, Government Doon Medical College, Dehradun, Uttarakhand, India \\ ${ }^{* *}$ Department of Physiology, AlIMS, Rishikesh, Uttarakhand, India \\ ${ }^{* * \star}$ Department of Physiology, Institute of Medical Sciences, Banaras Hindu University, Varanasi, India
}

\begin{abstract}
Introduction: Bisphenol A (BPA), an estrogenic compound is used in the manufacturing of plastics and also as coating for the inner linings of food packaging containers. It is reported to be an endocrine disruptor and produce toxicity in various organs. Since BPA exposure mainly occurs in the form of oral ingestion in humans, the present study was performed to examine the effects produced by the chronic exposure to BPA orally on kidney, liver and heart in rats.
\end{abstract}

Material \& Methods: Adult female rats of Charles Foster strain were used for the study. The rats were divided into two groups ( $\mathrm{n}=6$ in each group). In group 1, the rats were provided with food and water ad libitum whereas the rats of group 2 were fed with BPA containing pellets $(2 \mu \mathrm{g} / \mathrm{kg}$ body weight/day) for 30 days. Thereafter the heart, kidneys and liver were excised and processed for histopathological study.

Results: The histopathological examination of the organs in BPA fed rats showed major structural changes which manifested as loss of normal cytoarchitecture in all the three organs. Further there was significant reduction in the number of glomeruli in kidneys, degenerative changes in liver in the form of damage to portal triad and in heart the intercalated discs were damaged.

Conclusion: Chronic exposure to BPA by oral route produces renal toxicity as loss of glomeruli; hepatotoxicity as cytoarchitecture loss and cardiotoxicity as damage of intercalated disc changes.

Keywords: Histopathological examination, cytoarchitectural changes, BPA, lymphocytic infiltration.

\section{INTRODUCTION}

Bisphenol A (BPA), an endocrine disruptor is used in the manufacturing of poly epoxy resins and plastics. It is also used to coat the inner linings of food and beverage cans for preservation of edibles due to its fungicidal property [1]. BPA is a phenolic compound and is lipid soluble. It has been reported that BPA produces a number of toxic effects in experimental animals even at low doses [2-6]. BPA acts on estrogenic receptors and has been reported to produce numerous defects in experimental animals. These defects are manifested as infertility in both male and female mice [7,8], delayed onset of puberty [9], behavioural changes [10], predisposition to cancer in both male and female rats and mice [11], defects in growth and survival of neonatal mice [12] etc.

Further studies in human population have also shown the presence of BPA in the urinary samples of US and Chinese population [13-15]. Moreover studies have shown the co-relation of BPA levels with coronary heart diseases, liver defects and endocrine abnormalities in humans $[16,17]$. BPA can enter our body through various routes like oral ingestion, skin penetration, and nasal inhalation [18]. However, 
humans are mostly exposed to BPA through oral route by ingestion of food and beverages packed in plastic containers or cans. BPA ingested orally is absorbed from the intestine and is metabolised in liver. In liver, BPA is converted to BPA glucuronide which is water soluble and excreted through kidneys. However some of the free form of BPA which is not conjugated in liver is biologically active and being lipid soluble reaches various organs [19].

The dose of $<50 \mu \mathrm{g} / \mathrm{kg}$ bw/day is considered to be safe as per U.S. EPA (Environmental Protection Agency) [20]. However studies have reported toxic effects of BPA even at lower doses. In humans, the daily intake is reported to be within $50 \mu \mathrm{g} / \mathrm{kg} \mathrm{bw} / \mathrm{day}$. It was therefore hypothesized that BPA after oral ingestion may get accumulated in the organs with time and produce changes at the cellular level in various vital organs which might affect the functioning of the body. Hence the present study was undertaken to examine the effect of chronic exposure of BPA orally on the morphology of vital organs like heart, liver and kidneys in rats.

\section{MATERIAL AND METHODS}

The present study was performed on adult female Albino rats of Charles Foster strain weighing 150-200 grams after obtaining permission from Ethical Clearance Committee of the Institute of Medical Sciences, Banaras Hindu University, Varanasi, India.

The rats were kept in the animals room with temperature maintained at $25 \pm 0.5 \stackrel{\circ}{ } \mathrm{C}$, humidity $(50 \%$ of $\mathrm{RH}$ ) and light (12:12 hr light:dark). Food (Raj scientific Corporation, Varanasi) and water were provided ad libitum. The experiments were performed according to the guidelines given by Ethical Clearance Committee of the Institute of Medical Sciences, Banaras Hindu University, Varanasi, India. BPA was obtained from HiMedia Laboratory Pvt. Ltd. (Mumbai, India) and was dissolved in olive oil.

The animals were divided into two groups. In the first group $(n=6)$, the animals were allowed to drink tap water ad libitum for 30 days. In the second group $(n=6)$, the animals were fed with BPA filled food pellets $(2 \mu \mathrm{g} / \mathrm{kg} /$ day per animal; orally) for 30 days. After 30 days, the animals were killed using excessive dose of anaesthesia (urethane) and dissected. The heart, liver and kidneys were excised and preserved in formalin $(10 \%)$ for the study of histological changes.
Thereafter, the tissues were subjected to dehydration by exposing to increasing concentrations of alcohol $(70 \%-100 \%)$. Then the tissues were cleared and made translucent by placing them in xylol. This was followed by solidifying the tissues in molten paraffin and ribbon sections were made followed by slide formation. The slides prepared were subjected to Haematoxylin and Eosin stains. Further these slides were examined under microscope to observe for any histopathological changes.

\section{Statistical Analysis}

The semi-quantitative estimation of glomeruli was done by counting the number of glomeruli in 5 different fields and per field average was computed in both groups. All the data were pooled and mean \pm SEM were calculated. The data were compared by Student's t-test for unpaired observations. $p$ value < 0.05 was considered significant.

\section{RESULTS}

\section{Changes in kidneys}

In control group, normal cytoarchitecture of the nephrons showing numerous glomeruli in low power magnification (100x) was seen (Fig. 1a). Different diameter of tubules were also seen clearly. In higher magnification (400x), clear Bowman's capsule, vessels and healthy tubules were seen (Fig. 1b).
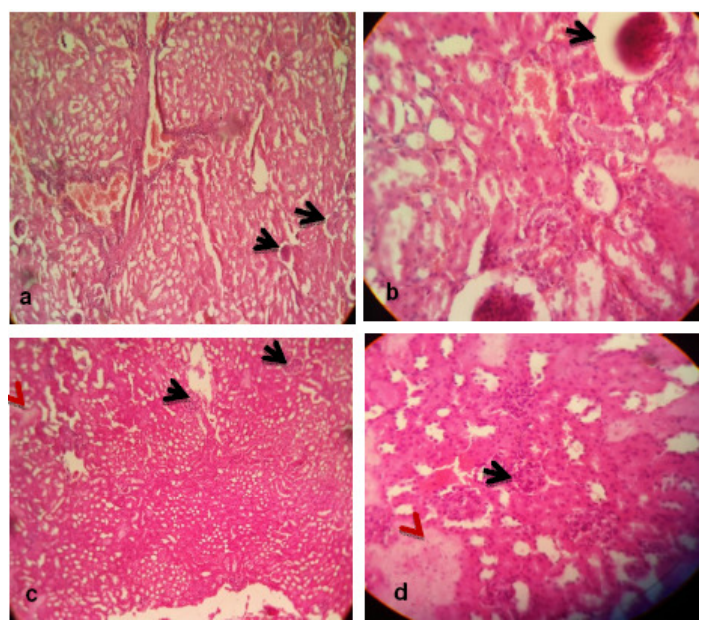

Fig. 1: Photomicrograph of HE stained kidney tissue of control rats and BPA treated rats. Normal cytoarchitecture is seen with presence of glomeruli (black arrows) in the control group (a\&b). In BPA fed rats (c\&d), loss of normal cytoarchitecture is seen. The glomeruli are less in number and the size of glomeruli is also reduced (black arrows). There are areas of hyaline deposits (red arrow). Magnification: 100x-a\&c, 400x-b\&d 
In BPA treated group, in low power the glomerular morphology was not clearly seen. The glomeruli appear small in comparison to the control group and there was even reduction in the number of glomeruli along with areas of hyaline deposits. Tubular architecture was also lost (Fig. 1C). At higher magnification, hyaline deposition was clearly seen with lymphocytic infiltration and there was significant shrinkage and distortion in the structure of glomeuli (Fig. 1d).

\section{Semi- quantitative estimation of the number of Glomeruli/field}

The histological changes in the kidneys demonstrated decrease in the number of glomeruli in BPA fed animals as seen in low power. Therefore, the semiquantitative estimation of glomeruli/field was done by counting the glomeruli in 5 different fields in both groups and then average value was taken to calculate per field number.

The observations revealed that treatment of the rats with BPA (2 $\mu \mathrm{g} / \mathrm{kg} /$ day) caused a significant decrease in the number of Glomeruli/field as compared to the time matched control group of rats $(p$ $<0.05$; Student's t-test for unpaired observations) (Fig. 2).

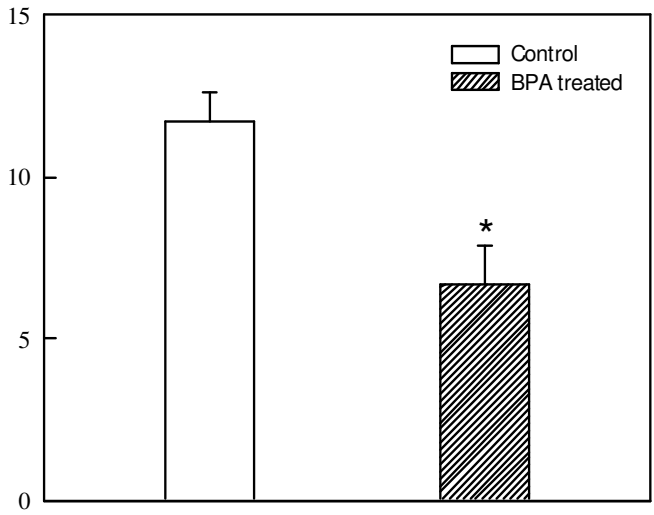

Fig. 2: Semi-quantitative estimation of gloweruli showing a significant reduction in the number of glomeruli in BPA fed rats. An asterisk represents $p<0.05$ as compared to control group (Student's t-test for unpaired observations)

\section{Changes in liver}

In control group, the normal laminar pattern with central vein and the hepatocytes were seen under both low and high power (Fig. 3a\&b). In case of BPA treated group, there was loss of the laminar pattern.
There were also areas showing distinct vacuoles (Fig. 3c\&d).
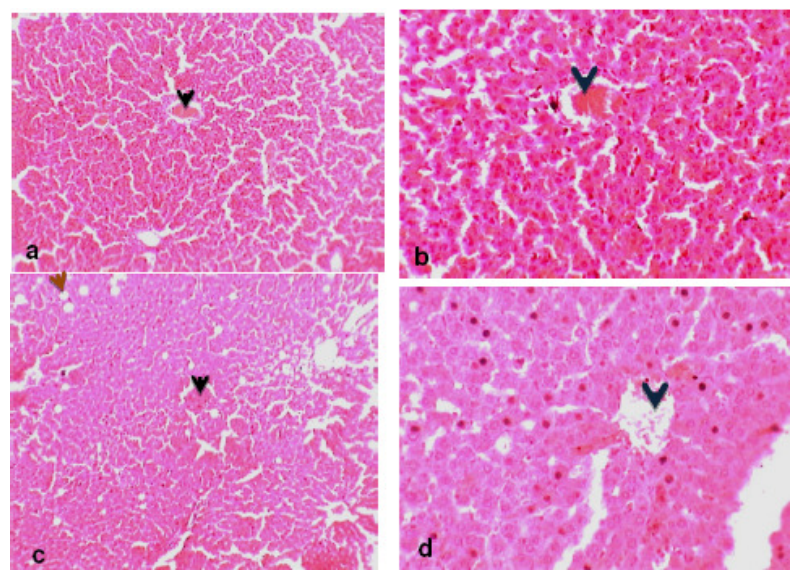

Fig. 3: Photomicrograph of HE stained liver tissue of control rats and BPA treated rats. Central vein (black arrow) and the normal laminar pattern was seen in control group (a\&b). In BPA fed group (c\&d), number of small vacuoles was seen (red arrow). Loss of laminar pattern was also observed. Magnification: 100x-a\&c, 400x-b\&d

\section{Changes in heart}

In control group, cardiac muscles with intercalated discs were seen (Fig. 4a). At higher magnification, striations with branched intercalated discs were seen (Fig. 4b). In the BPA treated group, broken myofibrils were seen (Fig. 4c\&d).
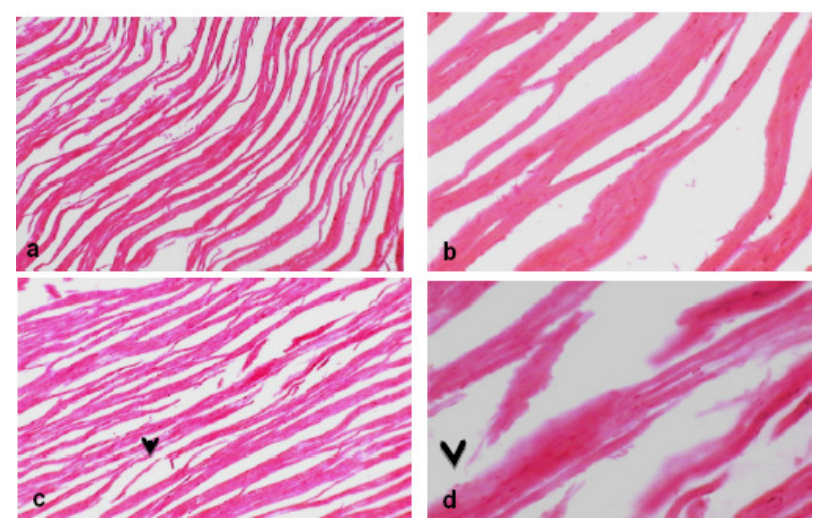

Fig. 4: Photomicrograph of HE stained heart tissue of control rats and BPA treated rats. Control group shows normal branching pattern (a\&b). In BPA fed group (c\&d), there is rupture of myofibrils (black arrow) and loss of intercalated discs. Magnification: 100x-a\&c, 400x-b\&d 


\section{DISCUSSION}

Chronic exposure in the form of oral ingestion of BPA (2 $\mu \mathrm{g} / \mathrm{kg} /$ day) for a period of 30 days produced extensive changes at organ level as seen on histological examination of heart, kidneys and liver. The dose selected for the present study was kept much lower than the recommended safe dose of $<50$ $\mu \mathrm{g} / \mathrm{kg}$ bw/day. Even this low dose of BPA produced cytoarchitectural changes in the vital organs in rats.

BPA after ingestion is known to be metabolised in liver by conjugation. The conjugated BPA is excreted out through the kidneys in the form of BPAglucuronide/sulphate. The excess concentration of BPA which is not metabolised is the free BPA which is active and toxic [20].

Taking into consideration this background knowledge of BPA metabolism, the possible mechanism responsible for the cytoarchitectural changes in the rats exposed to BPA in the present study was proposed. Probably, the ingested BPA was metabolised/ conjugated by liver and excreted by kidneys in these rats. However, the free unconjugated BPA, which is lipid soluble, might have accumulated in different organs due to daily exposure of these rats to BPA. This might BPA produced cytoarchitectural damage along with colloid deposition in liver and kidneys. There was significant reduction in the number and size of glomeruli in the BPA treated rats. The morphological damage produced in the nephrons by BPA exposure is likely to decrease the excretion of BPA thereby increasing the BPA load in the treated rats. Further, damage to the hepatocytes along with degenerative changes in liver produced by BPA reduced the metabolism/conjugation of BPA. Involvement of both the kidneys and liver is expected to increase the BPA (free) load as conjugation and excretion are decreased in the treated rats which in turn might have also produced histological changes in heart seen as loss of myofibrils and breakage of intercalated disks in BPA treated rats.

In the present study, all the morphological changes occurred after exposure to BPA at a very low dose of $2 \mu \mathrm{g} / \mathrm{kg} \mathrm{bw} / \mathrm{day}$. Exposure to BPA is increasing in the humans with increasing usage of plastic materials and packaged food and beverages. Even though the ingestion dose in humans is low, it is quite possible that this may lead to increased levels of free BPA which is toxic and may produce deleterious effects on the organs in humans similar to that in rats in the present study and lead to several diseases.

\section{CONFLICT OF INTERESTS}

The authors declare that they do not have any conflict of interests.

\section{REFERENCES}

1. Brotons JA, Olea-Serrano MF, Villalobos M, Pedraza V, Olea $\mathrm{N}$. Xenoestrogens released from lacquer coatings in food cans. Environmental Health Perspectives. 1995; 103: 608-612.

2. Kiguchi M, Fujita S, Lee J, Shimizu N, Koshikawa N. Behavioral responses to methylphenidate and apomorphine in rats exposed neonatally to bisphenol A. Journal of Oral Sciences. 2007; 49: 311-318.

3. Toufexis D. Region and sex specific modulation of anxiety behaviours in the rat. Journal of Neuroendocrinology. 2007; 19: 461-473.

4. Wetherill YB, Akingbemi BT, Kanno J, McLachan JA, Nadal A, Sonnenschein C, Watson CS, Zoeller RT, Belchar SM. In vitro molecular mechanisms of bisphenol A action. Reproductive Toxicology. 2007; 24: 178-198.

5. Wetherill YB, Petra CE, Monk KR, Puga A, Knudsen KE. The xenoestrogen bisphenol $A$ induces inappropriate androgen receptor activation and mitogenesis in prostate adenocarcinoma cells. Molecular Cancer Therapeutics. 2002; 1: $515-524$.

6. Zoeller RT, Bansal R, Parris C. Bisphenol-A, an environmental contaminant that acts as a thyroid hormone receptor antagonist in vitro, increases serum thyroxine, and alters RC3/neurogranin expression in the developing rat brain. Endocrinology. 2005; 146(2):607-12.

7. Sugiura-Ogasawara M, Ozaki Y, Sonta S, Makino T, Suzumori K. Exposure to bisphenol A is associated with recurrent miscarriage. Human Reproduction. 2005; 20: 2325-2329.

8. Yamasaki K, Sawaki M, Noda S, Imatanaka N, Takatsuki M. Subacute oral toxicity study of ethynylestradiol and bisphenol A, based on the draft protocol for the "Enhanced OECD Test Guideline no. 407”. Archives of Toxicology. 2002; 76: 65-74.

9. Tinwell H, Haseman J, Lefevre PA, Wallis N, Ashby J. Normal sexual development of two strains of rat exposed in utero to low doses of bisphenol A. Toxicological Sciences. 2002; 68: 339-348.

10. Patisaul HB, Fortino AE and Polston EK.. Differential disruption of nuclear volume and neuronal phenotype in the preoptic area by neonatal exposure to genistein and bisphenol $A$. Neurotoxicology. 2007; 28: 1-12.

11. Ho SM, Tang WY, Belmonte de Frausto J, Prins GS. Developmental exposure to estradiol and bisphenol A increases susceptibility to prostate carcinogenesis and epigenetically regulates phosphodiesterase type 4 variant 4 . Cancer Research. 2006; 66(11):1-9.

12. NTP. Bisphenol A: reproduction and fertility assessment in CD1 mice when administered in the feed. NTP 1985; 85-192. Research Triangle Park, NC. 
13. Calafat AM, Needham LL, Silva MJ. Lambert, G. Exposure to di-(2-ethylhexyl) phthalate among premature neonates in a neonatal intensive care unit. Pediatrics. 2004; 113: 429-434.

14. Calafat AM, Ye X, Wong LY, Reidy JA, Needham LL. Exposure of the U.S. population to bisphenol A and 4-tertiary-octylphenol: 2003-2004. Environmental Health Perspectives. 2008; 116: 3944.

15. He Y, Miao M, Herrinton LJ, Wu C, Yuan W, Zhou Z, Li DK. Bisphenol $A$ levels in blood and urine in a Chinese population and the personal factors affecting the levels. Environmental Research. 2009; 109(5): 629-33.

16. Lang IA, Galloway TS, Scarlett A, Henley WE, Depledge M, Wallace RB. Association of urinary bisphenol A concentration with medical disorders and laboratory abnormalities in adults. Journal of American Medical Association. 2008; 300: 13031310.
17. Melzer D, Rice NE, Lewis C, Henley WE, Galloway TS. Association of urinary bisphenol A with heart disease: evidence from NHANES 2003/06. 2010; PLoS ONE. 5(1): e8673.

18. Nisrin $K$, Catherine $H$, Henri $D$, Elizabeth $M$, Michel $P$. Cutaneous penetration of bisphenol $A$ in pig skin. Journal of toxicology and environmental health Part A. 2008; 71 (8): 4713.

19. Chapin R, Adams J, Boekelheide K, Gray L, Hayward S, Lees P, McIntyre B, Portier K, Schnorr T, Selevan S, Vandenbergh J, Woskie S. NTP-CERHR expert Panel Report on the reproductive and developmental toxicity of bisphenol A. 2008; Birth Defects Res B 83:157-395.

20. Zhuo $C$, Xuezhi Z, Dongliang $H$, Shibin D, Fangyi $X$, Huiqin $Y$, Xin J, Ying F, Li Y, Chong T, Chenjiang Y. Long-term exposure to a 'safe' dose of bisphenol A reduced protein acetylation in adult rat testes. Scientific Reports. 2017; 7: 40337. 